\title{
Vascular cognitive impairment risk among Mongolian adults: An overview
}

\author{
Darisuren Namjil ${ }^{1}$, Byambasuren Dagvajantsan ${ }^{1}$ and Oyuntugs Byambasukh ${ }^{2 *}$ \\ ${ }^{1}$ Department of Neurology, Mongolian National University of Medical Sciences, Ulaanbaatar 13270, Mongolia. \\ ${ }^{2}$ Department of Endocrinology, Mongolian National University of Medical Sciences, Ulaanbaatar 13270, \\ Mongolia. \\ * Correspondence: oyuntugs@mnums.edu.mn; Tel.: +976-99017063
}

Received: 4 July 2021; Accepted: 9 August 2021; Published: 16 September 2021

Edited by: Norshariza Nordin (Universiti Putra Malaysia, Malaysia)

Reviewed by: Buhari Ibrahim (Bauchi Sate University Gadau, Nigeria);

Usman Garkuwa (Bauchi Sate University Gadau, Nigeria)

https://doi.org/10.31117/neuroscirn.v4i3Suppl.99

\begin{abstract}
Cognitive impairment is commonly associated with older people. It can also occur in middle-aged people due to non-communicable diseases. The prevalence of lifestyle-related diseases (non-communicable diseases) has been rapidly increasing in Mongolia. Therefore, we aimed to overview these studies to identify whether the increasing prevalence of non-communicable disease is associated with the risks of cognitive impairment in Mongolians. Published literature between 01 January 1980 and 20 June 2021 were included in the study. We searched articles published in journals registered to PubMed and doctoral and master's dissertations registered in the Central Medical Library of Mongolia using the following keywords: "cognitive impairment", "dementia", "mild cognitive impairment", "Alzheimer", "vascular dementia", "diabetes", "Mongolia", "obesity", "stroke", "hypertension". While there were no internationally published articles in this field, seven studies were either published in local research journals or as doctoral or master's dissertations. Although few studies have been conducted in Mongolia, people with lifestyle-related conditions such as hypertension and diabetes are strongly associated with a higher risk of cognitive impairment. The increasing prevalence of non-communicable diseases may be one of the factors contributing to the prevalence of vascular cognitive impairment.
\end{abstract}

Keywords: Non-communicable diseases; cognitive impairment; vascular dementia;

(C2021 by Namjil et al. for use and distribution according to the Creative Commons Attribution (CC BY-NC 4.0) license (https://creativecommons.org/licenses/by-nc/4.0/), which permits unrestricted non-commercial use, distribution, and reproduction in any medium, provided the original author and source are credited.

\subsection{INTRODUCTION}

According to World Health Organization (WHO) reports, 50 million people have dementia worldwide (World Health Organization, 2019). Cognitive impairment (Cl) is a broad term including mild cognitive impairment (MCI) and dementia (Alzheimer's disease and others). $\mathrm{Cl}$ is caused by many risk factors, including ageing, lifestyle (risk factors for non-communicable diseases) and environmental factors (Bárrios et al., 2013; Mariani et al., 2007; World Health Organization, 2019). The increase in the ageing population may be a factor in the prevalence and incidence of $\mathrm{Cl}$; however, the rapid increase in non-communicable diseases, including diabetes mellitus (DM) and its risk factors, could contribute to the prevalence of vascular disease cognitive impairment. Studies showed that DM patients have a 2-4 times higher risk of cognitive impairment than non-DM people (Biessels et al., 2006). Furthermore, the increase of $\mathrm{Cl}$ can be mainly attributed to the rising number of those who have dementia in low- and middle-income countries, which is related to the increasing prevalence of non-communicable 
diseases in those countries (Biessels et al., 2006; Sharp et al., 2011).

In Mongolia, the prevalence of lifestyle-related diseases, considered non-communicable diseases, has been rapidly increasing for the last 30 years (World Health Organization, 2020). For instance, the prevalence of obesity was $17.3 \%$ in 1992 , and it has increased to 64\% in 2020 (World Health Organization, 2008, 2020). The prevalence of DM increased from 3.2\% in 1999 to $8.3 \%$ in 2020 (Suvd et al., 2002; World Health Organization, 2020). Stroke and cardiovascular mortality have been the leading cause of death, and Mongolia ranks third in the world in stroke-related deaths (World Life Expectancy, 2020). However, there is a lack of research on cognitive impairment among Mongolians. Therefore, we aimed to overview these studies to identify whether the increasing prevalence of the non-communicable disease is associated with the risks of cognitive impairment in Mongolians.

\subsection{LITERATURE SEARCH}

Due to the small population and the small number of researchers in our country, and the small number of studies conducted in any field of medicine, the possibility of conducting this research according to the systematic review methodology was limited. Thus, we have summarized the current research works in the area of $\mathrm{Cl}$. We searched for doctoral and master's dissertations registered in the Central Medical Library of Mongolia and articles published in the Mongolian Journal of Medicine. We also searched articles published in journals registered to PubMed using the following keywords: "cognitive impairment", "dementia", "mild cognitive impairment", "Alzheimer", and "vascular dementia". The information was collected from 01 January 1980 to 20 June, 2021. There were no internationally published articles in this field from Mongolia, seven studies conducted and published in local research journals and as doctoral or master's dissertations.

\subsection{COGNITIVE IMPAIRMENT (CI) IN MONGOLIAN ADULTS}

In a 2011 study by Oyunkhand on the prevalence of $\mathrm{Cl}$ in Mongolia, 39.7\% of Mongolian adults over 60 years old had $\mathrm{Cl}$, of which $59.5 \%$ had mild, $35.1 \%$ had moderate, and $5.4 \%$ had severe $\mathrm{Cl}$ (Oyunkhand, 2011). A study by Otgonbayar in 2017 conducted among the elderly in Ulaanbaatar showed that $27.0 \%$ had mild $\mathrm{Cl}$, and $5.0 \%$ had moderate $\mathrm{Cl}$. Approximately $10.7 \%$ of $\mathrm{Cl}$ occurred between 65 and 69 years, and $61.5 \%$ at $\geq 85$ years, indicating that $\mathrm{Cl}$ increases with age (Otgonbayar, 2017).

\subsection{VASCULAR COGNITIVE IMPAIRMENT (CI) IN MONGOLIAN ADULTS}

The first published study in the field of vascular $\mathrm{Cl}$ in Mongolia by Tserennadmid found that people with the cerebrovascular disease had a reduced memory when using a 10-word memorization test (Tserennadmid, 2005). Oyungerel (2018), who studied the prevalence of postoperative $\mathrm{Cl}$ in stroke patients, showed that $72.5 \%$ of them had $\mathrm{Cl}$. In the study, people with hypertension and stroke in strategic areas such as the anterior cerebral artery and left hemisphere were associated with $\mathrm{Cl}$ (Oyungerel, 2018). Enkhbold (2018) study indicated $4.6 \%$ mild $\mathrm{Cl}$ after cerebral artery microsurgery and $1.9 \%$ was severe $\mathrm{Cl}$ (Enkhbold, 2018). In 2012, a study by Khandsuren (2013) identified cognitive impairment in people with Parkinson's disease, and $27.3 \% \quad(n=13)$ of the patients were cognitively impaired (Khandsuren, 2013).

A study by Munkh-Undral (2021) compared people with and without type 2 DM using an age- and gendermatched case-control study (case $n=131$; control $n=131$ ) among those aged $40-80$ years old. They used Mini Mental Status Examination (MMSE) to identify mild $\mathrm{Cl}$ and evaluated the carotid artery carotid intima media thickness (cIMT) using Duplex/Doppler ultrasound. The MMSE scores were significantly different throughout study groups: $26.1 \pm 3.7$ and $27.5 \pm 2.6(p<0.001)$ for people with and without diabetes, respectively. DM patients have 3 times more risks of $\mathrm{Cl}$ when compared to people without DM. The cIMT was significantly different in DM $(1.72 \pm 0.97 \mathrm{~mm})$ and non-DM subjects $(1.21 \pm 0.60 \mathrm{~mm})$. Also, the resistance index of cIMT was statistically different in both groups. Mild $\mathrm{Cl}$ and cIMT were negatively correlated in DM subjects. The regression analysis suggests that diabetes duration and DM control might be the leading risk factor to develop $\mathrm{Cl}$ in diabetes (Munkh-Undral, 2021).

\subsection{DISCUSSION}

This study aimed to get an overview of relevant research works to identify whether the increasing prevalence of non-communicable diseases is associated with the increased risks of $\mathrm{Cl}$ in Mongolian adults. However, there is a lack of research on $\mathrm{Cl}$. We found only seven studies either published in local research journals or as doctoral or master's dissertations. There were no internationally published articles in this field. Two of these studies determined the prevalence of $\mathrm{Cl}$ in the general population, but they did not link $\mathrm{Cl}$ to lifestyle- 
related risk factors. The remaining five attempted to link the risk of cerebrovascular disease (also lifestyle-related risk factors) to $\mathrm{Cl}$.

Due to the lifestyle changes of Mongolians, especially urbanization, the prevalence of non-communicable diseases (lifestyle-related diseases) such as obesity, hypertension and diabetes has dramatically increased over the last 30 years (World Health Organization, 2020). For instance, before 1990, diabetes and hypertension were rare among Mongolians, but from 1990 to 2020, the prevalence of obesity was 3.5 times higher, the prevalence of diabetes was 2.5 times higher, and the prevalence of hypertension was twice as high (World Health Organization, 2020). Furthermore, the prevalence of $\mathrm{Cl}$ in the general population at that time and before was not studied. The first prevalence study was only conducted in 2011 (Oyunkhand, 2011). In addition, that study did not identify risk factors of $\mathrm{Cl}$ with a wide range. Since then, no population-based surveys have been conducted to determine the prevalence and risk factors for cognitive impairment. This made it impossible for us to study how risk factors of $\mathrm{Cl}$ are changing, nor was it possible to assess whether the spread of $\mathrm{Cl}$ increased or decreased in the general populations. Therefore, further studies are needed to identify the prevalence of $\mathrm{Cl}$ among the general population. These should also determine the risk factors of $\mathrm{Cl}$.

In addition to non-modifiable risk factors such as age and genetic predisposition, there has been considerable research works in recent years on modifiable risk factors that may increase the risk of $\mathrm{Cl}$ (World Health Organization, 2019). Studies found that lifestyle-related risk factors such as physical inactivity, unhealthy diets and harmful use of alcohol and tobacco contribute to cognitive impairment and dementia (World Health Organization, 2019). For instance, according to metaanalysis based studies conducted in China, the prevalence of $\mathrm{Cl}$ has increased rapidly from 8.5 to $21.8 \%$ in the last 20 years in that country. Physical inactivity was identified as one of the reasons for this (Deng et al.,
2021). Furthermore, review studies concluded that certain medical conditions, including hypertension, obesity and diabetes, are associated with increased risk of dementia (Biessels et al., 2006; Sharp et al., 2011). Therefore, in 2017, the World Health Assembly endorsed the Global Action Plan on the public health response to dementia 2017-2025 which includes several guidelines for interventions to reduce $\mathrm{Cl}$ (World Health Organization, 2019).

A recent study in Mongolia found that the risk of mild $\mathrm{Cl}$ in people with diabetes is three times higher than in people without diabetes (Munkh-Undral, 2021). Diabetes duration and diabetes control are the main risk factors in the development of cognitive impairment in people with diabetes. Also, the carotid intima-media thickness and resistance index were higher in diabetic patients than in the non-DM group. It was also associated with cognitive impairment, suggesting that carotid shift findings indicate macro- and micro-vascular complications; it may be a predictor of cognitive impairment in diabetes (Munkh-Undral, 2021). This is similar to the results found in the abovementioned review and shows that people with diabetes have a higher risk of mild Cl (Biessels et al., 2006).

\subsection{CONCLUSIONS}

Based on the studies conducted in Mongolia, people with hypertension and diabetes have a higher risk of cognitive impairment. Therefore, the increasing prevalence of non-communicable diseases may be one of the factors contributing to the prevalence of vascular cognitive impairment. There is a need to study in detail the risk factors of cognitive impairment among Mongolian adults in the future.

Author Contributions: $\mathrm{OB}$ and $\mathrm{BD}$ conceived and designed the experiments; $\mathrm{DN}, \mathrm{OB}$, and $\mathrm{BD}$ analyzed the data; $O B$ and $D N$ wrote the paper; $O B$ and $B D$ revived and edited the paper.

Conflicts of Interest: The authors declare no conflict of interest.

\section{References}

Bárrios, H., Narciso, S., Guerreiro, M., Maroco, J., Logsdon, R., \& de Mendonça, A. (2013). Quality of life in patients with mild cognitive impairment. Aging \& Mental Health, 17(3), 287-292. https://doi.org/10.1080/13607863.2012.747083

Biessels, G., Staekenborg, S., Brunner, E., Brayne, C., \& Scheltens, P. (2006). Risk of dementia in diabetes mellitus: a systematic review. The Lancet Neurology, 5(1), 64-74. https://doi.org/10.1016/S1474-4422(05)70284-2

Deng, Y., Zhao, S., Cheng, G., Yang, J., Li, B., Xu, K., Xiao, P., Li, W., \& Rong, S. (2021). The Prevalence of Mild Cognitive Impairment among Chinese People: A Meta-Analysis. Neuroepidemiology, 55(2), 79-91.

https://doi.org/10.1159/000512597 
Enkhbold, G. (2018). The results of the microsurgery in arterial aneurysma of anterior cerebral circulation. Mongolian National University of Medical Sciences.

Khandsuren, B. (2013). Predictors of cognitive impairment in Parkinson's disease. Mongolian National University of Medical Sciences.

Mariani, E., Monastero, R., \& Mecocci, P. (2007). Mild Cognitive Impairment: A Systematic Review. Journal of Alzheimer's Disease, 12(1), 23-35. https://doi.org/10.3233/JAD-2007-12104

Munkh-Undral, M. (2021). A study of cognitive impairment in people with type 2 diabetes in relation to changes in carotid intima-media thinckness. Mongolian National University of Medical Sciences.

Otgonbayar, L. (2017). Mild cognitive impairment in Elderly people in Ulaanbaatar. Mongolian Neurology Journal, 1(18), 4447.

Oyungerel, T. (2018). Evaluation of the repeatable battery for the assessment of neuropsychologigal status (RBANS) in screening for cognitive impairment following stroke. Mongolian National University of Medical Sciences.

Oyunkhand, R. (2011). A study on Mongolian elderly people. Mongolian National University of Medical Sciences.

Sharp, S. I., Aarsland, D., Day, S., Sønnesyn, H., \& Ballard, C. (2011). Hypertension is a potential risk factor for vascular dementia: systematic review. International Journal of Geriatric Psychiatry, 26(7), 661-669. https://doi.org/10.1002/GPS.2572

Suvd, J., Gerel, B., Otgooloi, H., Purevsuren, D., Zolzaya, H., Roglic, G., \& King, H. (2002). Glucose intolerance and associated factors in Mongolia: results of a national survey. Diabetic Medicine, 19(6), 502-508. https://doi.org/10.1046/J.14645491.2002.00737.X

Tserennadmid, B. (2005). Result of transcranial doppler sonography studies on elderly people. Mongolian National University of Medical Sciences.

World Health Organization. (2008). Mongolian STEPS Survey on the prevalence of noncommunicable disease risk factors 2006. https://www.who.int/ncds/surveillance/steps/December 2006 Mongolia STEPS Survey.pdf

World Health Organization. (2019). Risk Reduction of Cognitive Decline and Dementia. In Risk Reduction of Cognitive Decline and Dementia: WHO Guidelines. World Health Organization. https://www.ncbi.nlm.nih.gov/books/NBK542796/

World Health Organization. (2020). Mongolia completes its 4th round of STEPS survey on the prevalence of NCD risk factors. https://www.who.int/mongolia/news/detail/29-06-2020-mongolia-completes-its-4th-round-of-steps-survey-on-theprevalence-of-ncd-risk-factors

World Life Expectancy. (2020). Stroke in Mongolia. https://www.worldlifeexpectancy.com/mongolia-stroke 